\title{
Practical Measuring method of Thickness for Zinc-Coating in Steel Transmission Towers
}

\author{
Shi-jun Huang ${ }^{1, a}$, Hai-feng Zhu ${ }^{1, b}$, Tian-wen Wang ${ }^{2, \mathrm{c}}$ and Hao Chen ${ }^{2, \mathrm{~d}}$ \\ ${ }^{1}$ State Grid Jiangsu Econgomic Research Institute, Nanjing Jiangsu 210008,China \\ ${ }^{2}$ School of civil engineering, wuhan university, Hubei Wuhan 430072, China \\ a994757079@qq.com, b2316272845@qq.com, \\ c1643224011@qq.com, d302881574@qq.com
}

\begin{abstract}
Keywords: practical measuring method; zinc-coating; steel comparison; steel transmission towers Abstract. The thickness of zinc-coating thickness was a parameter of significant importance on steel transmission towers. When the zinc-coating's thickness does not meet the required standard, the tower's steel members were easily to be corroded. However most current detection methods focusing only on the total thickness of steel coatings, and the thickness of zinc-coating can hardly be measured by existing methods. This paper presents the experimental analysis of different methods of zinc-coating's thickness' measurement. Comparison was conducted and the most accurate and practical measuring method was proposed.
\end{abstract}

\section{Introduction}

Due to corrosion, the steel zinc-coating in steel transmission towers will get thinner and even fall off from steel member surfaces, during the long term usage of the steel structures. Without the protection of zinc-coating, the steel in the towers were very easy to be oxidated, and when the corrosion process began, the steel member will soon lose its weight and the effective section of the steel members will decrease. Thus the thickness of steel members' zinc-coating becomes a very important parameter to predicate the reliability of the exposed steel members.

However the existing measuring methods of zinc-coating's thickness were not accurate enough and the methods were difficult to be conducted. Durden Jr etc. [1] studied the corrosion of transmission towers' foundations and provided the solution for the foundation corrosion, but study on zinc-coating thickness on the upper steel structures was not conducted. Fang etc. [2] studied the Zinc coating thickness quality control of angle steel tower materials based on statistical method, but the measurement was not mentioned. Liu and Zhang [3] investigated the inspecting method for the zinc-coating quality for steel support of hydrol gic cable, but the thickness of zinc-coating was not fully discussed. In a word, current measure methods for thickness of steel coating were available only when testing the total thickness. However, steel coating usually contains not only zinc-coating but coat of painting and other layers, and zinc-coating can hardly be separated during the measurement.

This paper presents the experimental analysis of different methods of zinc-coating's thickness' measurement. By Comparing the results of different measure methods, the most accurate and practical measuring method was proposed.

\section{Experimental process}

\section{Experimental procedure}

The reduction method was employed in the study process. The galvanization and coating applying process were conducted. And when each layer was applied, the thickness of total steel coating was measured and recorded at each time.

The measurement of the steel coating was conducting using a coating thickness gauge, which has a magnetic head putting on the steel surface when measuring, as steel was a magnetic substance material, the non-magnetic thickness can be read by the coating thickness gauge. 
It can be seen from how the gauge works that, the thickness of each different layer can hardly be measured accurately. Considering this, the coating was cleared by different means in the tests, and the thickness of the remaining coating was measured in the last step.

\section{Measurement arrangement}

The steel members were chosen as angle steels. The surfaces of the angle steel were divided into inter-surface and outer-surface two groups, and each group was further divided into several regions, as illustrated in fig. 1. Thicknesses of coating on each region were measured five times in different points, and the average value from each region was calculated and recorded. The reduction process of each group can be described as

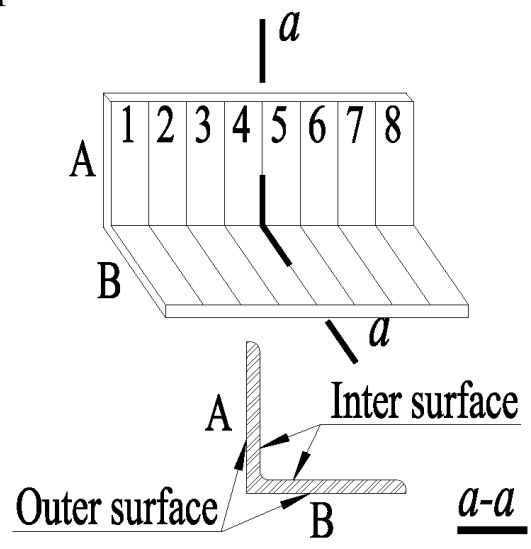

Fig.1 Tested angle steel

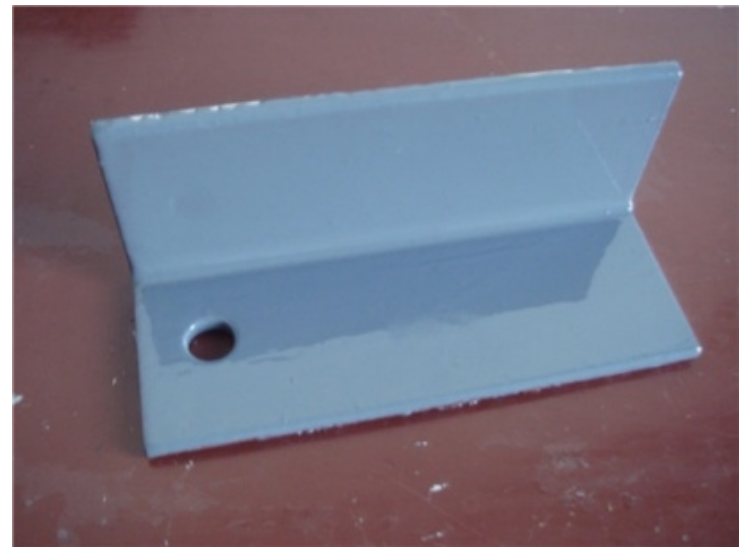

Fig.2 Angle steel surface of alkyd varnish layer

1) Group 1 (inter-surface)

Firstly (step 1), measure the total thickness of the steel coating just after the galvanization was completed, and the thickness was defined as $\mathrm{t}_{11}$. Secondly (step 2), apply antirust paint on the steel surface, and measure the total coating thickness $\mathrm{t}_{12}$. Thirdly (step 3), apply grey alkyd varnish on the basis of the former layers, as shown in Fig.2, and then measure and record the total coating thickness $t_{13}$. Finally (step 4), use different methods to clean up coatings from step 2 and step 3 , then record the remaining coating thickness $\mathrm{t}_{1 \mathrm{r}}$.

2) Group 2 (outer-surface)

The process steps was similar to that of group 1, with the coatings in step 2 and step 3 were two layers of the same aluminium paint.

It should be noted that, all the layers applied on the steel surface were common coatings can be seen in real steel transmission towers. And the efforts to achieve the most accurate and practical measuring method, were focusing on finding best method to wash up different steel coating layers while keep zinc-coating not being affected. The one which make $t_{1 r}\left(t_{2 r}\right)$ most near $t_{1}\left(t_{2}\right)$, was the deserved measuring method.

\section{Experimental results}

Results from step 1 and surface groups were summarized in Table 1.

Table 1 Results from step 1

\begin{tabular}{|c|c|c|c|c|c|c|c|c|c|c|}
\hline \multicolumn{2}{|c|}{ Group No. } & \multicolumn{8}{|c|}{ Initial measured zinc-coating thickness $/ \mu \mathrm{m}$} & \multirow{2}{*}{$\frac{\text { Mean value } / \mu \mathrm{m}}{117}$} \\
\hline \multirow{2}{*}{$1 t_{11}$} & A & 116 & 119 & 112 & 118 & 119 & 124 & 113 & 116 & \\
\hline & $\mathrm{B}$ & 112 & 115 & 114 & 110 & 117 & 120 & 112 & 110 & 114 \\
\hline \multirow{2}{*}{$2 t_{21}$} & A & 128 & 133 & 132 & 126 & 128 & 123 & 122 & 122 & 127 \\
\hline & $\mathrm{B}$ & 123 & 124 & 128 & 125 & 125 & 130 & 124 & 125 & 126 \\
\hline
\end{tabular}


Results from step 2 and surface groups were summarized in Table 2.

Table 2 Results from step 2

\begin{tabular}{|c|c|c|c|c|c|c|c|c|c|c|}
\hline \multicolumn{2}{|c|}{ Group No. } & \multicolumn{8}{|c|}{ Total thickness for step $2 / \mu \mathrm{m}$} & \multirow{2}{*}{$\frac{\text { Mean value } / \mu \mathrm{m}}{163}$} \\
\hline \multirow{2}{*}{$1 t_{12}$} & A & 159 & 160 & 160 & 164 & 169 & 164 & 159 & 168 & \\
\hline & B & 176 & 180 & 181 & 179 & 176 & 178 & 184 & 183 & 180 \\
\hline \multirow{2}{*}{$2 t_{22}$} & A & 129 & 139 & 144 & 143 & 145 & 147 & 134 & 152 & 142 \\
\hline & B & 134 & 135 & 137 & 140 & 145 & 143 & 138 & 142 & 139 \\
\hline
\end{tabular}

Results from step 3 and surface groups were summarized in Table 3.

Table 3 Results from step 3

\begin{tabular}{|c|c|c|c|c|c|c|c|c|c|c|}
\hline \multicolumn{2}{|c|}{ Group No. } & \multicolumn{8}{|c|}{ Total thickness for step $3 / \mu \mathrm{m}$} & \multirow{2}{*}{$\frac{\text { Mean value } / \mu \mathrm{m}}{213.9}$} \\
\hline & $\mathrm{A}$ & 219 & 204 & 221 & 222 & 199 & 193 & 224 & 229 & \\
\hline $1 \iota_{13}$ & $\mathrm{~B}$ & 239 & 245 & 238 & 239 & 241 & 236 & 241 & 239 & 239.8 \\
\hline \multirow{2}{*}{$2 t_{23}$} & $\mathrm{~A}$ & 157 & 164 & 158 & 162 & 160 & 164 & 163 & 166 & 161.8 \\
\hline & B & 155 & 160 & 161 & 154 & 161 & 161 & 157 & 158 & 158.4 \\
\hline
\end{tabular}

Results from step 4 and surface groups were summarized in Table 4.

In step 4, there were total three different ways to clean up the outer coatings, including: sanding, washing surfaces by paint remover, and washing surfaces by isoamyl acetate.

Table 4 Results from step 4

\begin{tabular}{|c|c|c|c|c|c|c|c|c|c|c|c|c|}
\hline \multirow{2}{*}{\multicolumn{2}{|c|}{ Group No. }} & \multicolumn{11}{|c|}{ Total thickness for step $4 / \mu \mathrm{m}$} \\
\hline & & \multicolumn{2}{|c|}{ Sanding } & \multirow{2}{*}{$\frac{\text { Mean }}{153}$} & \multicolumn{3}{|c|}{ Paint remover } & \multirow{2}{*}{$\frac{\text { Mean }}{180}$} & \multicolumn{3}{|c|}{ Isoamyl acetate } & \multirow{2}{*}{$\frac{\text { Mean }}{119}$} \\
\hline \multirow{2}{*}{$1 t_{1 \mathrm{r}}$} & A & 137 & 169 & & 180 & 178 & 181 & & 123 & 116 & 117 & \\
\hline & B & 162 & 127 & 144 & 171 & 126 & 169 & 155 & 123 & 122 & 111 & 118 \\
\hline \multirow{2}{*}{$2 t_{2 \mathrm{r}}$} & A & 136 & 148 & 142 & 152 & 147 & 130 & 143 & 126 & 127 & 129 & 127 \\
\hline & B & 125 & 143 & 134 & 156 & 132 & 136 & 141 & 138 & 132 & 125 & 132 \\
\hline
\end{tabular}

The results from step 4 were compared with results from step 1. And the comparison was summarized in Table5.

Table 5 Comparison error results

\begin{tabular}{|c|c|c|c|c|}
\hline \multicolumn{2}{|c|}{ Group No. } & $\begin{array}{c}\text { Sanding } / \% \\
\mathrm{t}_{1 \mathrm{r}} / \mathrm{t}_{11}-1 \\
\left(\mathrm{t}_{2 \mathrm{r}} / \mathrm{t}_{21}-1\right) \\
\end{array}$ & $\begin{array}{c}\text { Paint remover } / \% \\
\mathrm{t}_{1 \mathrm{r}} / \mathrm{t}_{11}-1 \\
\left(\mathrm{t}_{2 \mathrm{r}} / \mathrm{t}_{21}-1\right) \\
\end{array}$ & $\begin{array}{c}\text { Isoamyl acetate } / \% \\
\mathrm{t}_{11} / \mathrm{t}_{11}-1 \\
\left(\mathrm{t}_{2 \mathrm{r}} / \mathrm{t}_{21}-1\right) \\
\end{array}$ \\
\hline \multirow{2}{*}{$1 t_{1 r}$} & A & 31.0 & 53.6 & 1.3 \\
\hline & B & 26.5 & 36.2 & 3.9 \\
\hline \multirow{2}{*}{$2 t_{2 r}$} & A & 11.9 & 12.7 & 0.3 \\
\hline & B & 6.5 & 12.1 & 4.7 \\
\hline \multicolumn{2}{|l|}{ Mean } & 19.0 & 28.6 & 2.6 \\
\hline \multicolumn{2}{|c|}{ Standard deviation } & 11.7 & 20.0 & 2.1 \\
\hline
\end{tabular}

It can be seen from Table 5 that, the way of washing surfaces by isoamyl acetate was the most accurate mean to provide pure zinc-coating, and measurement after isoamyl acetate washing was the closest value to the initial thickness value of zinc-coating, with $2.6 \%$ higher on average, and the standard deviation was 2.1. The isoamyl acetate washing process was easy to conduct, and most 
importantly, zinc-coating was affected little by the washing process, thus the isoamyl acetate washing measurement for zinc-coating thickness was practical and accurate enough, which also meet the standard of nondestructive examination.

Besides, it should be noted that, these findings can also be employed in measurement of thickness for zinc-coating on I members, box members and any other type of steel members.

\section{Conclusion}

Through experimental tests and comparisons, the following conclusions were made:

1) The current coating-detection method was only available when measuring total coating thickness, while the thickness of zinc-coating can hardly be obtained.

2) The method of washing off outer coating layers was proved to be a practical and accurate way to obtain the thickness of zinc-coating.

3) Isoamyl acetate was proved to be the most suitable reagent to be used in the washing process, as which can clear outer coating completely with little effects on zinc-coating.

\section{References}

[1] Durden Jr., Harry, Maddox, Jonathan. Unique solution for $230 \mathrm{kV}$ transmission tower grillage foundation corrosion. Electrical Transmission and Substation Structures. 2012, 239-249.

[2] Fang Ji-tao, Wang Hui, Deng Wei-wei. Zinc coating thickness quality control of angle steel tower materials based on statistical method. Journal of Theoretical and Applied Information Technology, $46(2), 812-818$.

[3] Qiang Liu, Hong-ping Zhang. Discuss on the inspecting method for the zinc coating quality of the steel tower units for the hydrologic cable. Journal of Water Resources \& Water Engineering. 2006, 17(5), 93-94. 